\section{New treatment opportunities for patients with advanced and/or metastatic thyroid cancer}

According to the RARECAREnet definition [1], thyroid carcinomas (TCs) are rare malignant tumors being their crude incidence rate in Europe of 5.07/100,000/year (CI 95\% 5.03-5.10).TCs included three different tumor types: i) differentiated thyroid carcinoma (DTC), which derives from the follicular thyroid cells including papillary (PTC) and follicular carcinomas (FTC) and their variants ii) medullary thyroid carcinoma (MTC) originating from the parafollicular C cell and considered a neuroendocrine carcinoma, and iii) anaplastic thyroid carcinoma (ATC). This latter has an epithelial origin and may evolve from a pre-existing DTC (most commonly papillary phenotype) or originate de novo.

Surgery (emi- or total thyroidectomy) is the standard of care in each histotype, combined with radioactive iodine (RAI) only in high-risk DTC cases. Prognosis is related to the histotype: better in case of DTC (5-yr relative survival 91-96\%) and MTC (5-yr relative survival 88\%) while definitely worse for ATC (5-yr relative survival 14\%) [2]. Outcome drops down dramatically at the appearance of distant metastases and in case of RAI-resistant DTC, being $30 \%$ at 5 years for these latter subjects [3] and $40 \%$ at 10 years for MTC patients [4].

Except RAI for DTC patients, no other effective treatment was available for patients with relapsed and/or metastatic TC since few years ago. Doxorubicin was approved on 1974 and since that date no new drugs have been approved. The presence of several genetic aberrations both in DTC and in MTC such as gene rearrangements (RET/PTC; PAX8 PPAR $\gamma$; RET/NTRK1; etc.) and mutations (RET M918T; BRAFV600E; $R A S$; etc.) paves the way to test new compounds as multi-tyrosine kinase inhibitors (TKIs). Moreover, TCs are characterized by a high intrinsic vascularization and VEGFR is tailored by most of TKIs under evaluation in TCs.

In the last 10 years, several compounds have been investigated (e.g. motesanib, sunitinib, axitinib, pazopanib), most within phase II while only some of them in phase III trials. This is the case of sorafenib [5] and lenvatinib in RAI-resistant DTC [6] and vandetanib [7] and cabozantinib in MTC [8]. Primary aim was met in each trial, demonstrating a significant advantage in progression-free survival in the treatment arm in comparison with placebo although no conclusive data on overall survival have been published yet. Based on these trials, all latter 4 drugs were approved for patients with relapsed and/or metastatic TCs by the international regulatory agencies as Food and Drug Administration and European Medicine Agencies: vandetanib was the first approved drug on 2011, followed by cabozantinib (2012), sorafenib (2013) and lenvatinib

\section{Correspondence to:}

Laura Locati,

SC Oncologia Medica 3, Tumori

della Testa e Collo, Fondazione IRCCS, Istituto Nazionale dei Tumori, Via Venezian 1, 20133 Milano, Italy. Phone: +3902 23902805

Fax: +3902 23903769

E-mail: laura.locati@istitutotumori.mi.it CANCER BREAKING NEWS 2017;5(3):3-5 DOI: $10.19156 / \mathrm{cbn} .2017 .0051$ 
(2015). The authorization of these agents addressed a dramatic unmet need for patients with relapsed and/or metastatic RAI-resistant DTC and MTC, representing a real therapeutic innovation in this setting of patients.

Although these drugs are currently authorized in many countries, reimbursement is not provided by all the national health systems, so the treatment options for these patients remain still scant. For example, in Italy sorafenib and cabozantinib are currently approved but not reimbursed while lenvatinib and vandetanib are the only therapeutic option for RAI-resistant DTC and MTC patients, respectively. Some issues need to be still addressed. For example, how to reduce the toxicities burden that currently involves the majority of treated patients as well as the lack of a clear benefit in terms of overall survival. Even if a significant survival improvement has been recently reported within two subgroups of patients: subjects with RAI-resistant DTC older than 65 years on lenvatinib [9] and patients with metastatic MTC carrying RETM918T mutation on cabozantinib [10].

Moreover, some questions about the efficacy are still open: which type of patients could get a real benefit from these drugs? When is the right moment to start a TKIs? The approved dose is the right one? Some trials are already ongoing to try to address these questions. Unfortunately, despite the major developments of the last years in the field of genetic profile and therapeutic management in TCs, no progress has been done in ATC that still remains a neglected disease with a very dismal prognosis.

Laura D. Locati, Salvatore Alfieri, Lisa Licitra Head and Neck Medical Oncology Unit Fondazione IRCCS Istituto Nazionale dei Tumori, Milan, Italy

\section{Conflicts of Interest}

LDL acted as consultant or advisory board member for the EISAI; LL acted as consultant or advisory board member for the following companies: EISAI and Bayer.

\section{References}

1. Gatta G, Capocaccia R, Botta L et al. Burden and centralised treatment in Europe of rare tumours: results of RARECAREnet-a population-based study. Lancet Oncol 2017;18(8):1022-39.

2. Dal Maso L, Tavilla A, Pacini F et al. Survival of 86,690 patients with thyroid cancer: A population-based study in 29 European countries from EUROCARE-5. Eur J Cancer 2017;77:140-52.

3. Durante C, Haddy N, Baudin E et al. Long-term outcome of 444 patients with distant metastases from papillary and follicular thyroid carcinoma: benefits and limits of radioio- dine therapy. J Clin Endocrinol Metab 2006;91(8):2892-9.

4. Sippel RS, Kunnimalaiyaan M, Chen H. Current management of medullary thyroid cancer. Oncologist 2008;13(5):539-47.

5. Brose MS, Nutting CM, Jarzab B et al. Sorafenib in radioactive iodine-refractory, locally advanced or metastatic differentiated thyroid cancer: a randomised, doubleblind, phase 3 trial. Lancet 2014;384(9940):319-28.

6. Schlumberger M, Tahara M, Wirth LJ et al. Lenvatinib versus placebo in radioiodine-refractory thyroid cancer. N Engl J Med 2015;372(7):621-30. 
7. Wells SA Jr, Robinson BG, Gagel RF et al. Vandetanib in patients with locally advanced or metastatic medullary thyroid cancer: a randomized, double-blind phase III trial. J Clin Oncol 2012;30(2):134-41.

8. Elisei R, Schlumberger MJ, Müller SP et al. Cabozantinib in progressive medullary thyroid cancer. J Clin Oncol 2013;31(29):3639-46.

9. Brose MS, Worden FP, Newbold KL et al. Effect of age on the efficacy and safety of lenvatinib in radioiodinerefractory differentiated thyroid cancer in the phase III SELECT Trial. J Clin Oncol 2017;35(23):2692-9.

10. Schlumberger M, Elisei R, Müller $S$ et al. Overall survival analysis of EXAM, a phase III trial of cabozantinib in patients with radiographically progressive medullary thyroid carcinoma. Ann Oncol 2017;28(11): 2813-9. 